and have non-p-integral invariants. The proof is due to Serre, and it is supplemented by the author to include also the case where the curves are defined over a function field (of characteristic zero), and their invariants are transcendental. Finally, the field of division points of an elliptic curve $A$ over an algebraic number field $K$ is studied. Let $A_{0}$ denote the group of division points of $A$ (= points of finite order), and $K\left(A_{0}\right)$ the field generated by their coordinates over $K$. If $A$ has no complex multiplication, it is known from Serre's work that the Galois group of $K\left(A_{0}\right)$ over $K$ is an open subgroup of the product $\Pi_{p} \mathrm{GL}_{2}\left(Z_{p}\right)$ (taken over all primes $p$ ). This important theorem is proved here under the additional assumption that the invariant of $A$ is nonintegral.

Part Four enters into the multiplicative theory of elliptic (theta) functions, and the connection to $L$-series. After first dealing with the analytic theory, exhibiting the classical multiplicative functions and their formulas, the author defines the so-called Siegel functions, which are certain integral modular functions. Their singular values lie in certain well-defined ray class fields; their behavior under Galois automorphisms is deduced from the general reciprocity law of Shimura mentioned above. Two Kronecker limit formulas involving (multiplicative) elliptic functions are established, as well as their relation to $L$ series over imaginary quadratic number fields. In particular, their value at $s=$ 1 is worked out, which plays an important role in algebraic number theory in connection e.g. with class number formulae.

The attentive reader who has travelled up to this point over the ocean of elliptic functions, with this book as his vessel and the author as his guide, will certainly be able to sail further on his own to the scenes of great discoveries past and present. The tour is to be highly recommended even though the sea sometimes may be going rough.

Peter Roquette

\title{
BULLETIN OF THE
}

AMERICAN MATHEMATICAL SOCIETY

Volume 82, Number 4, July 1976

Maximal orders, by I. Reiner, Academic Press, London, New York, San Francisco, 1975, xii + 395 pp., \$36.50.

The theory of orders is a fascinating and difficult subject which occupies much of the common ground between algebra and number theory. Since this theory is known to relatively few contemporary mathematicians, I will give a more than usually thorough survey of the general area, before discussing the book itself.

To facilitate the discussion, we start with the definition and a few examples. Let $R$ be a Dedekind domain (that is, $R$ is a Noetherian integral domain in which all nonzero prime ideals are maximal, and which is integrally closed in its field of fractions $K$. The last condition means that any element of $K$ which is a zero of a monic polynomial with coefficients in $R$ belongs to $R$. As examples, one may take principal ideal domains and the rings of algebraic integers of algebraic number fields). An $R$-order is intended to be a certain type of $R$-algebra. Some of the examples which should be included are the ring of all $n \times n$ matrices over $R$ (for any $n \geqslant 1$ ), the group ring $R G$ of a finite 
group whose order is a unit of $K$, and the integral closure of $R$ in a finite separable extension field $L$ of $K$. In each of these cases, we see that the given $R$-algebra is a finitely generated torsionfree $R$-module, and the $K$-algebra obtained from it is quite well behaved. The precise definition abstracts all this, starting from the last condition first. As a basic context, we take a finitedimensional, separable $K$-algebra $A$, where "separable" means that $L \otimes_{K} A$ is semisimple for all extension fields $L$ of $K$. An $R$-order in $A$ is a subring $\Lambda$ (with $1)$ of $A$ such that $\Lambda$ is a finitely generated $R$-module which spans $A$ as a $K$ vector space. $\Lambda$ is a maximal order if it is contained in no larger $R$-order in $A$. In the motivating examples above, the ring of $n \times n$ matrices is a maximal $R$ order in the algebra of $n \times n$ matrices over $K$; the integral closure of $R$ in $L$ is the unique maximal $R$-order in $L$. (In general, if $A$ is a commutative separable $K$-algebra, then the integral closure of $R$ therein is the unique maximal $R$ order in $A$.) The group ring $R G$ is an $R$-order in the group algebra $K G$, but is maximal if and only if the order of $G$ is a unit of $R$.

To the best of my knowledge, the term "order" was first used by Dedekind in the 1870's. There were at least two distinct problems that motivated him. On the one hand, Kummer, in unpublished work, had purported to prove Fermat's Last Theorem, by using factorizations in rings of cyclotomic integers. Unfortunately, Kummer assumed that such factorizations were unique. That this is not true was soon discovered, and Kummer himself attempted to restore unique factorization by adjoining "ideal elements". (These are elements of extension fields whose adjunction kills off elements of the ideal class group.) Dedekind's insight here was to observe that the ideals (in the sense we use the term now) of the cyclotomic rings factor uniquely as products of prime ideals. This knowledge is sufficient to prove Fermat's Last Theorem for exponents which are regular primes. (It is easy to see that in discussing Fermat's Last Theorem, one need only consider exponents that are odd primes, once the case of exponent four is settled.) Dedekind did not confine his attention here to cyclotomic rings. Indeed, he showed that the unique factorization property holds for ideals in the rings of algebraic integers of arbitrary algebraic number fields. A crucial aspect of the discussion is that one must use the ring of all algebraic integers in the number field to achieve uniqueness. Hence, for example, one must work in $\mathbf{Z}[(1+\sqrt{-3}) / 2]$ in order to obtain a satisfactory arithmetic in $Q(\sqrt{-3})$. Dedekind's predecessors had rejected such quantities as $(1+\sqrt{-3}) / 2$ as integers, because of the presence of the denominator, as well as for technical reasons arising from the theory of integral quadratic forms. However, $(1+\sqrt{-3}) / 2$ is acceptably integral because it satisfies the monic polynomial equation $x^{2}-x+1=0$, with integer coefficients. Hence, we may say that Dedekind recognized the importance of maximal orders in the discussion of arithmetic.

On the other hand, Dedekind also had occasion to discuss nonmaximal orders in number fields. In his Disquisitiones arithmeticae, Gauss gave a lengthy and beautiful discussion of integral binary quadratic forms (note however that he allowed only forms $Q(x, y)=A x^{2}+B x y+C y^{2}$ with $B$ even). One of the most interesting aspects of the discussion is the notion of composition of forms. This notion allows one to work on Diophantine equations $Q(x, y)=m$ by working primarily on the case where $m$ is prime (the 
genus problem). The point is to multiply forms so as to obtain formulas of the type

$$
Q_{1}(x, y) Q_{2}(z, w)=Q_{3}(\alpha, \beta),
$$

where $\alpha$ and $\beta$ are integral bilinear functions of $x, y, z$ and $w$. Such formulae generally exist only when $Q_{1}$ and $Q_{2}$ have the same discriminant. Dedekind observed that equivalence classes of forms of given discriminant correspond to classes of invertible ideals of a nonmaximal order in a quadratic number field, and that under this correspondence, composition of forms is carried over to multiplication of ideals. This observation was cast in very general form by Kaplansky in the late 1960's.

For about forty years after these fundamental papers of Dedekind appeared, work on orders dealt almost exclusively with algebraic number theory, i.e., with the theory of maximal orders in global fields. Profound advances were made by many mathematicians; among them we mention Dedekind, Kronecker, Weber, Hilbert, Hensel, Hecke, Landau, and Minkowski. Since there are many excellent accounts available of this era, we will skip ahead to 1916, when Brandt used orders in generalized quaternion algebras to discuss composition of quaternary quadratic forms in the same style used by Dedekind for the binary case. (We remark that for the quaternary forms, one does not use the ordinary norm, which is quartic, but rather the quadratic reduced norm.) The introduction of orders in noncommutative algebras significantly raises the level of difficulty. The main source of extra complexity is that the integral elements of a noncommutative algebra need not form a ring. Hence, there is no strict analogue of the ring of all algebraic integers of a number field. One can still show that maximal orders exist (in separable algebras), but a given algebra may contain many of them. Because of this sort of difficulty, it is often convenient to discuss fractional ideals of all maximal orders in a given algebra at once. This point of view was emphasized in Brandt's work, and led him to the discovery of the Brandt groupoid of an algebra. This groupoid is roughly analogous to the group of fractional ideals of a Dedekind ring.

The 1920's and 1930's were a sort of a golden age of the theory of maximal orders. The most significant work dealt with multiplicative ideal theory and finiteness of class number (in various senses). We mention that Artin, Speiser, Dickson, Deuring, Eichler, and Chevalley were among those who made important contributions. We single out for special attention a profound and beautiful calculation by Hasse of maximal orders in division algebras over fields that are complete with respect to discrete valuations. He showed that such division algebras behave much like local fields; to wit, the valuation of the ground field extends uniquely to a valuation (in a suitable sense) of the division algebra. The valuation ring is then the unique maximal order. In case the residue field of the original ground domain is finite, one can exhibit the skewfield as the crossed product corresponding to a cyclic, unramified extension of the ground field. These special features permit the calculation of the Brauer groups of the local and global fields of number theory, which in turn leads to the central results of classfield theory. The connection with classfield theory is explained in Weil's austere but elegant Basic number theory. 
Activity in the general theory of orders diminished considerably in the forties and fifties (although algebraic number theory of course continued to flourish). In 1960, the time was ripe for modern homological methods to take their place in the theory of orders. The basic work along these lines was done by Auslander and Goldman. They gave homological characterizations of maximal orders in several different settings. Their work led Strooker to discover that an order is maximal if and only if it is hereditary and clean. Cleanness can be defined in several equivalent ways; one definition is that the reduced special projective class group is finite.

In recent years, attention has focused on nonmaximal orders, especially from a representation-theoretic viewpoint. There have been at least three major directions: group rings, hereditary orders, and orders of finite representation type. The representations of integral group rings have significant applications in several branches of mathematics. We mention Wall's homotopy and surgery obstructions in topology, the calculation of space groups and fundamental groups of the flat manifolds in geometry, and the discussion of integral normal bases in algebraic number theory. Further, integral group rings provide the vital link between ordinary and modular representations of finite groups. The interest in hereditary orders comes from the work of Auslander, Goldman, and Strooker mentioned above. Thorough discussions have now been given by Harada, Brumer, Jacobinski, and Reiner. An $R$ order $\Lambda$ has finite representation type if there are but finitely many indecomposable finitely generated, $R$-torsionfree $\Lambda$-modules. The interest in these orders parallels that in Artinian rings of finite representation type, a subject which has seen dramatic developments recently. A thorough discussion of these orders can be found in Roggenkamp's excellent Springer-Verlag Lecture Notes. I would also like to point out that orders play a role in connecting algebraic geometry with classfield theory. To each complex elliptic curve admitting complex multiplication there corresponds an ideal class in a nonmaximal $\mathbf{Z}$-order in an imaginary quadratic field. The order determines the isogeny class of the curve; the ideal class determines the isomorphism class. The geometric and analytic invariants of the curve in turn lead to an explicit description of the Hilbert classfield of the quadratic field. In all, we see that the theory of orders is an active one which has much to contribute to many parts of mathematics.

One might ask why we need a book on maximal orders, when the above discussion indicates that nonmaximal orders dominate the current areas of research. The answer is that maximal orders play an indispensable role in the general theory of orders. Typically, one embeds a given order $\Lambda$ in a maximal order $\Lambda^{\prime}$. The two become equal when localized at almost all primes of the ground ring, so results about $\Lambda^{\prime}$ often yield much information about $\Lambda$ itself. Dedekind used this approach in his earliest work on nonmaximal orders in number fields; profound investigations of Jacobinski show that the method is equally powerful for noncommutative orders.

Reiner's book gives by far the most extensive and most readable account available of the classical theory of maximal orders. After an introductory chapter that recalls necessary background material from ring theory, the structure theory for maximal orders is developed in the natural fashion. First, 
Hasse's work on orders in complete skewfields is presented. An interlude on Morita equivalence permits passage to the case of maximal orders in separable algebras over local fields. Classical techniques then transfer the information thus far derived to the case of maximal orders over arbitrary Dedekind rings. At each stage, ideal theory, different, discriminant, and norms are discussed in full detail.

The latter part of the book is devoted to some interesting special topics in the theory of orders. The theory of Brauer groups is developed from the point of view of crossed products, and, in particular, of cyclic algebras. This discussion is very thorough, and would provide all the background necessary for the applications to classfield theory. The only important results asserted without proof are the Hasse Norm Theorem and the Grundwald-Wang Theorem.

Other topics covered are Eichler's Theorem on reduced norms, hereditary orders and some of the recent results of the author, Ullom, Frölich, and others on class groups and Picard groups of orders.

The book has been written with great care, and is a pleasure to read. Unlike many books at such an advanced level, it contains many interesting exercises, with hints where appropriate; it contains almost no misprints or mistakes. It is essential to the library of every working algebraist and number theorist.

W. H. Gustafson

BULLETIN OF THE

AMERICAN MATHEMATICAL SOCIETY

Volume 82, Number 4, July 1976

The Stone-Čech compactification, by Russell C. Walker, Ergebnisse der Mathematik und ihrer Grenzgebiete, Band 83, Springer-Verlag, New York, 1974, $\mathrm{x}+332$ pp., $\$ 30.40$.

Stone and Čech published their papers on $\beta X$, the "Stone-Čech compactification" of $X$, in 1937. Here $X$ is a completely regular Hausdorff space. The space $\beta X$ is characterized as the maximal compactification of $X$ : every mapping from $X$ to a compact space $K$ extends to a mapping from $\beta X$ to $K$. It is sufficient to state this for $K \subset \mathbf{R}: \beta X$ is that compactification of $X$ in which $X$ is $C^{*}$-embedded, i.e., every bounded continuous real-valued function on $X$ extends to $\beta X$.

With the existence and uniqueness of $\beta X$ thus established, from one point of view the subject is closed. From another point of view it has just been opened. What are particular properties of $\beta X$ and how do they reflect properties of $X$ ? Čech raised some specific questions, and answered some of them, in his original paper. For instance, $\beta X$ is connected if and only if $X$ is connected; on the other hand, $\beta X$ is never metrizable (for noncompact $X$ ). What about $X^{*}$, i.e., $\beta X \backslash X$ ? What does $\mathrm{N}^{*}$, for example, "really look like?" What is its cardinal number, for that matter? Pospiśil answered this last question in a note published side by side with Čech's paper: for any infinite discrete space $D,|\beta D|=\exp \exp |D|$ and hence $\left|D^{*}\right|=\exp \exp |D|$; in particular, $\left|\mathbf{N}^{*}\right|=2^{\text {c }}$. (The year before, Hausdorff had solved the same problem but in a form not recognized at the time as equivalent.)

The next major development was the 1939 paper of Gelfand and Kolmogo- 